\title{
Correction to: Validation of the modified Skåne emergency department assessment of patient load (mSEAL) model for emergency department crowding and comparison with international models; an observational study
}

\author{
Jens Wretborn ${ }^{1,2^{*}}$, Håkan Starkenberg ${ }^{3,4}$, Thoralph Ruge ${ }^{5,6}$, Daniel B. Wilhelms $s^{1,7}$ and Ulf Ekelund ${ }^{2}$
}

BMC Emerg Med 21, 21 (2021)

https://doi.org/10.1186/s12873-021-00414-6

It was highlighted that in the original article [1] the Ethics approval and consent to participate section was incorrect. This Correction article shows the incorrect and correct Ethics approval and consent to participate section. The original article has been updated.

Incorrect

Ethics approval and consent to participate.

The study was approved by the regional ethics committé at Linköping University and Karolinska institutet.

\section{Correct}

Ethics approval and consent to participate.

The part of the study pertaining to the Linköping University Hospital and Motala Community Hospital was approved by the regional ethics committee at the county of Östergötland. The data from the Karolinska Hospital in Huddinge and Solna was part of a quality improvement initiative only including anonymised data, and as such is exempt from ethical approval in Sweden.
The original article can be found online at https://doi.org/10.1186/s12873021-00414-6.

* Correspondence: jens.wretborn@med.lu.se

'Department of Emergency Medicine, Local Health Care Services in Central Östergötland, Linköping, Östergötland, Sweden

${ }^{2}$ Department of Clinical Sciences Lund, Emergency Medicine, Faculty of Medicine, Lund University, Lund, Sweden

Full list of author information is available at the end of the article

\begin{abstract}
Author details
'Department of Emergency Medicine, Local Health Care Services in Central Östergötland, Linköping, Östergötland, Sweden. ${ }^{2}$ Department of Clinical Sciences Lund, Emergency Medicine, Faculty of Medicine, Lund University, Lund, Sweden. ${ }^{3}$ Enköping Hospital, Region Uppsala, Sweden. ${ }^{4}$ Department of Emergency Medicine Solna, Karolinska Institutet, Solna, Sweden. ${ }^{5}$ Department of Emergency and Internal Medicine, Skåne University Hospital, Malmö, Sweden. ${ }^{6}$ Department of Clinical Sciences Malmö, Faculty of Medicine, Lund University, Lund, Sweden. ${ }^{7}$ Department of Biomedical and Clinical Sciences, Faculty of Health Sciences, Linköping University, Linköping, Sweden.
\end{abstract}

Published online: 16 April 2021

Reference

1. Wretborn, et al. Validation of the modified Skåne emergency department assessment of patient load (MSEAL) model for emergency department crowding and comparison with international models; an observational study. BMC Emerg Med. 2021;21:21. https://doi.org/10.1186/s12873-02100414-6.

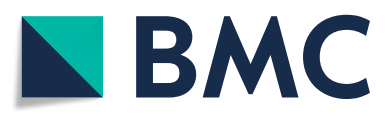

(c) The Author(s). 2021 Open Access This article is licensed under a Creative Commons Attribution 4.0 International License, which permits use, sharing, adaptation, distribution and reproduction in any medium or format, as long as you give appropriate credit to the original author(s) and the source, provide a link to the Creative Commons licence, and indicate if changes were made. The images or other third party material in this article are included in the article's Creative Commons licence, unless indicated otherwise in a credit line to the material. If material is not included in the article's Creative Commons licence and your intended use is not permitted by statutory regulation or exceeds the permitted use, you will need to obtain permission directly from the copyright holder. To view a copy of this licence, visit http://creativecommons.org/licenses/by/4.0/ The Creative Commons Public Domain Dedication waiver (http://creativecommons.org/publicdomain/zero/1.0/) applies to the data made available in this article, unless otherwise stated in a credit line to the data. 